\title{
Técnica de Masquelet en no unión atrófica de tibia con osteomielitis crónica, su descripción
}

\author{
Masquelet technique in atrophic nonunion of tibia with \\ chronic osteomyelitis, its description
}

\author{
Francisco Adolfo De los Santos Montoya, * José Alonso Ceballos Sánchez,* \\ Erick Hernández Frías,* Mauricio Sierra Pérez,* Zazhil Valeria Herrera Caballero*
}

Citar como: De los Santos MFA, Ceballos SJA, Hernández FE, Sierra PM, Herrera CZV. Técnica de Masquelet en no unión atrófica de tibia con osteomielitis crónica, su descripción. Acta Med Grupo Angeles. 2021; 19 (2): 280-284. https://dx.doi.org/10.35366/100456

\section{Resumen}

La técnica por inducción de membranas o técnica de Masquelet ha sido desarrollada en los últimos años para el tratamiento de defectos óseos, ya sea traumáticos o infecciosos con adecuada cobertura de tejidos blandos, por medio de un procedimiento dividido en dos fases, en donde con cemento quirúrgico se estimula la creación de tegumentos en el foco de no unión o de pérdida ósea con posterior colocación de injerto. Se presenta un caso en donde se realiza el procedimiento antes descrito, con buenos logros funcionales en el tratamiento de no unión atrófica con osteomielitis en paciente con fractura de tibia diafisaria.

Palabras clave: Masquelet, pseudoartrosis, osteomielitis, inducción, membranas.

\section{INTRODUCCIÓN}

Desde la publicación del artículo original en 2003 por Masquelet y colaboradores, la técnica de inducción de membranas ha tenido una gran aceptación y desarrollo de alcance internacional, con un porcentaje de 67 a 100\% de unión ${ }^{1}$ en cirugías bien indicadas para esta mecánica, describiéndose hasta $25 \mathrm{~cm}$ de pérdida ósea con adecuados resultados. ${ }^{2}$

\footnotetext{
* Servicio de Traumatología y Ortopedia, Nuevo Sanatorio Durango, Facultad Mexicana de Medicina, Universidad La Salle.
}

Correspondencia:

Francisco Adolfo de los Santos Montoya Correo electrónico:

delossantosmontoyaadolfo@gmail.com

Aceptado: 27-08-2020.

www.medigraphic.com/actamedica

\section{Abstract}

The membrane induction technique or Masquelet technique has been developed in recent years for the treatment of either traumatic or infectious bone defects with adequate soft tissue coverage, by means of a procedure divided in two phases, where the creation of membranes is stimulated with surgical cement in the focus of non-union or bone loss with subsequent graft placement, a case is presented where the technique described above is performed with good functional results in the treatment of an atrophic non-union with osteomyelitis in a patient with a fractured diaphyseal tibia.

Keywords: Masquelet, pseudoarthrosis, osteomyelitis, induced, membranes.

Tal pérdida de hueso es un reto dentro de la ortopedia, ya que la desvitalización de los fragmentos genera un escenario complicado al buscar la fusión de un tejido óseo, ya sea infectado o fragmentado por un traumatismo, sin embargo, durante la descripción original de la norma se pudo observar el gran potencial osteogénico del procedimiento en dos fases, siendo la primera el desbridamiento del foco óseo y la colocación de un espaciador de cemento quirúrgico, persiguiendo la creación de membranas alrededor del meollo de la fractura, y la segunda fase consistente en el retiro del aglomerante y el emplazamiento del injerto ya sea autólogo o heterólogo. ${ }^{3}$

A continuación, se describe la técnica utilizada en una paciente con no unión atrófica por fractura de tibia simple diafisaria con osteomielitis.

\section{CASO CLÍNICO}

En el Centro Hospitalario Nuevo Sanatorio Durango, en el Servicio de Ortopedia, se recibe a paciente de 
sexo femenino, de 32 años de edad, quien fue víctima de atropellamiento por vehículo automotor, con herida cortante en región de la pierna izquierda, de $9 \mathrm{~cm}$ de longitud por $1 \mathrm{~cm}$ de ancho, que conecta con planos profundos y que expone el foco de fractura diafisaria de tibia y peroné izquierdos, cuenta con antecedente de fractura expuesta de tibia en la misma pierna hace 12 años, tratada con fijación externa y posterior enclavado centromedular, con desarrollo de osteomielitis actualmente no activa, se inicia administración de antibiótico intravenoso con cefalotina de tercera generación y aminoglucósidos, se coloca férula muslopodálica con fibra de vidrio y se toma serie traumática de estudios de imagen así como proyecciones AP y lateral de la pierna izquierda, se le ingresa con diagnóstico de fractura expuesta de tercio medio de tibia y peroné izquierdos $\mathrm{AO}$ 42A3, clasificación Gustilo Anderson grado II, Tscherne Oestern grado 2.

Seis horas después se le ingresa a quirófano para aseo quirúrgico, desbridamiento, toma de cultivo, de biopsia y colocación de fijador externo unilateral, monoplanar a tibia izquierda; con cultivos negativos, se realiza siete días después el enclavado con clavo centromedular de tibia canulado, más ubicación de matriz ósea e injerto alogénico en foco de fractura, se le da de alta y se decide continuar el seguimiento por consulta externa.

Después de nueve meses se observa no unión de espectro atrófico en tibia y peroné izquierdos (Figura 1) por lo que se decide su internamiento para realización de técnica de Masquelet, se informa a la paciente acerca del pronóstico y posibles múltiples procedimientos.

Se realiza la técnica de inducción de membranas, debridando foco de fractura, con $2 \mathrm{~cm}$ de defecto óseo en diáfisis tibial, se toman cultivos y biopsia de hueso y se coloca un espaciador de cemento quirúrgico medicado (Figura 2).

Ocho semanas posteriores, con cultivos negativos, en radiografía se observa fatiga del primer perno distal del clavo centromedular así como una apropiada posición del espaciador en tibia izquierda, por lo cual se resuelve continuar con el segundo paso del proceso, se retira dicho elemento cuidando la membrana perifocal, se emplaza injerto autólogo de cresta iliaca, heterólogo y matriz ósea en foco de fractura, cuidando una cobertura adecuada de las corticales (Figura 3).

A las cuatro semanas posteriores se realiza el seguimiento sin datos de complicaciones postquirúrgicas, con cita a tal efecto en cuatro semanas con radiografía AP y lateral de pierna, en donde se observa una conveniente colocación de injerto, se realizan citas de verificación cada dos meses con radiografías de control; a los seis meses se perciben datos de consolidación acorde a la técnica, integrando puentes óseos en al menos tres corticales y exhibiendo marcha sin dolor (Figura 4), por lo que se inicia marcha asistida con bota larga tipo walker que deriva tolerada, la cual se retira a los dos meses.

En la actualidad es capaz de marcha asistida con andadera de cuatro puntos, sin dolor a la misma.

\section{DISCUSIÓN}

La técnica de inducción de membranas se ha reportado con una tasa de éxito mayor de $90 \%$, con un promedio de afianzamiento de ocho meses y con un promedio de $6 \mathrm{~cm}$ en la extensión del defecto, aun con un $30 \%$ de prevalencia de infecciones óseas. ${ }^{4}$

Inclusive con quebrantos asociados a alta energía o con gran exposición de fragmentos de hueso, como pueden ser los tipos III A, B y C en la clasificación de Gustilo Anderson modificada, se obtuvo una consolidación en el $100 \%$ de los pacientes, con resultados funcionales buenos y con marcha independiente. ${ }^{5}$

Todavía no es un método del todo aceptado por los cirujanos ortopedistas a nivel internacional, argumentándose altas tasas de refractura e infección, en comparación con la técnica de transporte óseo con llizarov, empero, la práctica de Masquelet tiene mejores logros en cuanto a fusión, aunque es de recalcar que también cuenta con más complicaciones en cuanto a infección y riesgo de amputación, de acuerdo al metaanálisis de Meng y colegas. ${ }^{6}$

En cuanto a otras técnicas históricas, como peroné vascularizado, cuenta con mejores resultados funcionales y porcentaje de consolidación en miembros pélvicos, ya que no se recomienda el uso en miembros pélvicos de peroné
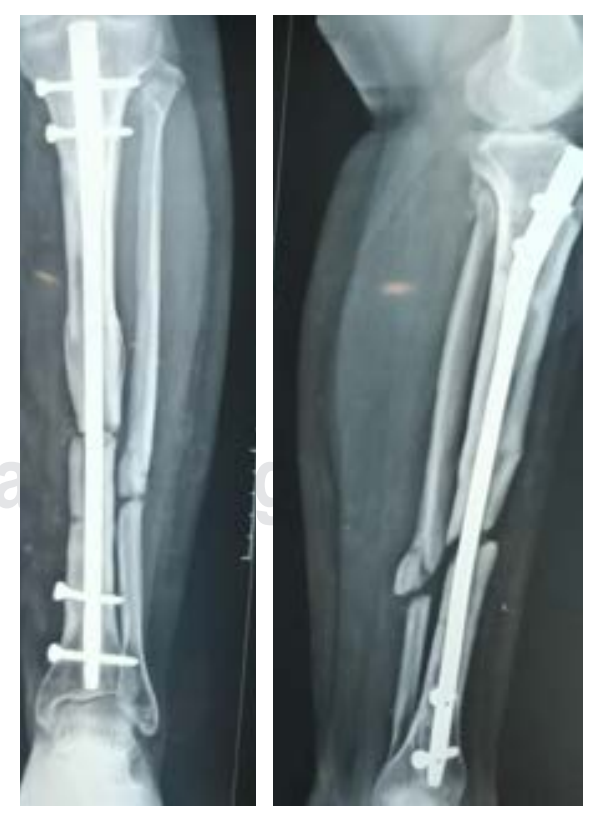

Figura 1:

Radiografía a los nueve meses posteriores a la primera cirugía, con colocación de calvo centro medular, sin datos de consolidación. 


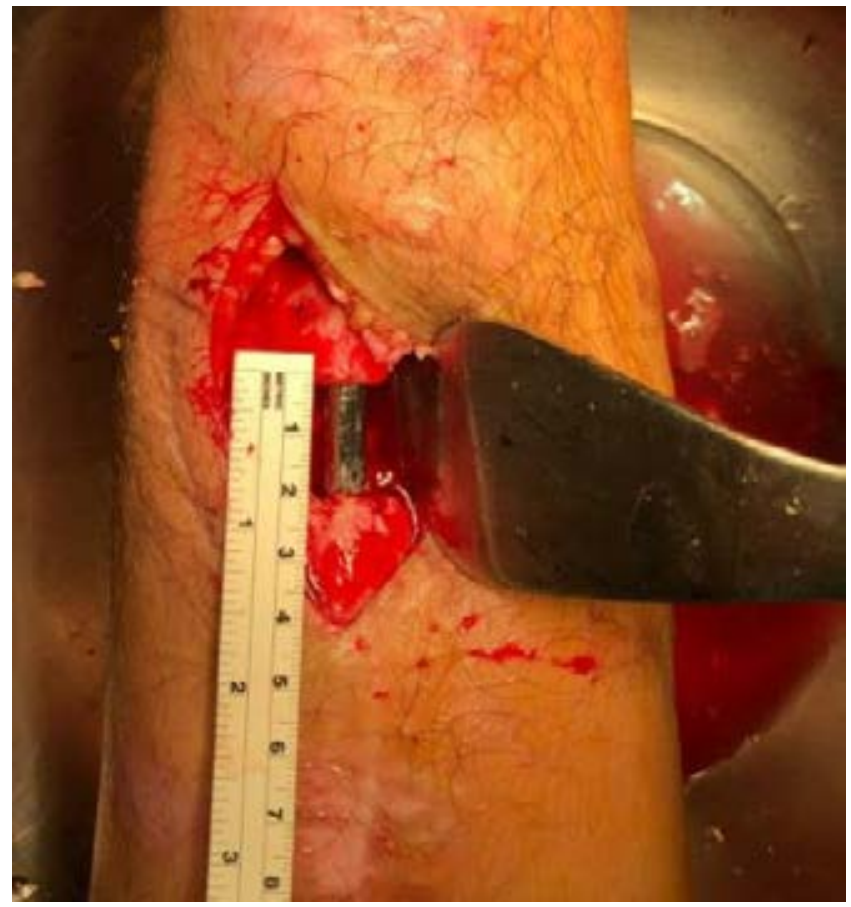

Figura 2: Foto transquirúrgica en la que se observa defecto óseo de $2 \mathrm{~cm}$.

vascularizado, muchos autores reservan esta operación para las extremidades torácicas, sobre todo en radio y cúbito teniendo altos porcentajes de fractura vistos en tibia proximal, además de ser una práctica más costosa y más exigente en cuanto al desarrollo de la misma, requiriendo equipo especializado y personal familiarizado con la microcirugía; por otro lado, la colocación de injerto autólogo rinde buenos frutos en miembros pélvicos, sin embargo, ante la lesión de circulación endóstica y perióstica que conlleva la osteomielitis crónica, no se recomienda su uso si el sitio de recepción no cuenta con adecuada irrigación del área siendo frecuentes las infecciones ante estos escenarios. ${ }^{7,8}$

Indicaciones. Su uso es muy variado, desde defectos óseos postraumáticos sépticos y asépticos, todo el espectro de no unión, resecciones óseas por tumores y hasta medicina de guerra, podría utilizarse en niños y adultos, siempre y cuando tengan una cobertura ósea adecuada y se estabilice la parte ósea, puede ser en defectos agudos o crónicos, en cualquier segmento del hueso tanto metafisario como diafisario excepto articulación, y es una técnica viable para virtualmente cualquier hueso largo. ${ }^{1,9}$

Contraindicaciones. Al ser una práctica relativamente nueva, no hay muchos estudios de seguimiento a largo plazo por lo que las contraindicaciones aún no son muy variadas; éstas incluyen: defectos sin apropiada cobertura de tejidos blandos o demasiado lesionados en donde no es viable una reconstrucción, defectos articulares y tegumento expuesto a radioterapia con posterior resección de tumor. ${ }^{1}$

\section{Elección de implante}

Aunque en la técnica original sólo se menciona la estabilización ósea del defecto, los cirujanos se han inclinado a dos vertientes, la fijación interna y la externa, siendo mejor la estabilización interna ya que se configura un adecuado canal, así como un verdadero estrés a la cortical, por la descarga en el sitio de aplicación de injerto.

La literatura apoya la idea del uso del clavo intramedular con descarga temprana a fin de mejorar resultados de unión y función. Con una evidencia de nivel III, 121 personas fueron sometidas a la misma técnica de inducción de membranas (56 tibias y 65 fémures); con la diferencia del uso de implante, en un grupo se decidió el uso de clavo intramedular y en el otro el uso de placa no especificando su tipo.

El primer grupo, en el cual se utilizó el clavo, tuvo un acortamiento en el tiempo procurando permitir al paciente la descarga (2.4 versus 4.6 meses), en comparación con el otro grupo, en donde se utilizó la placa y se logró una disminución en el riesgo de reoperación (17 versus 46\%), por lo que se indica que, siempre y cuando sea viable, la utilización de fijación interna con clavo centromedular en la propuesta de Masquelet, en tibia y fémur, se prefiera este tipo de implante frente a la estabilización con placa. ${ }^{10}$

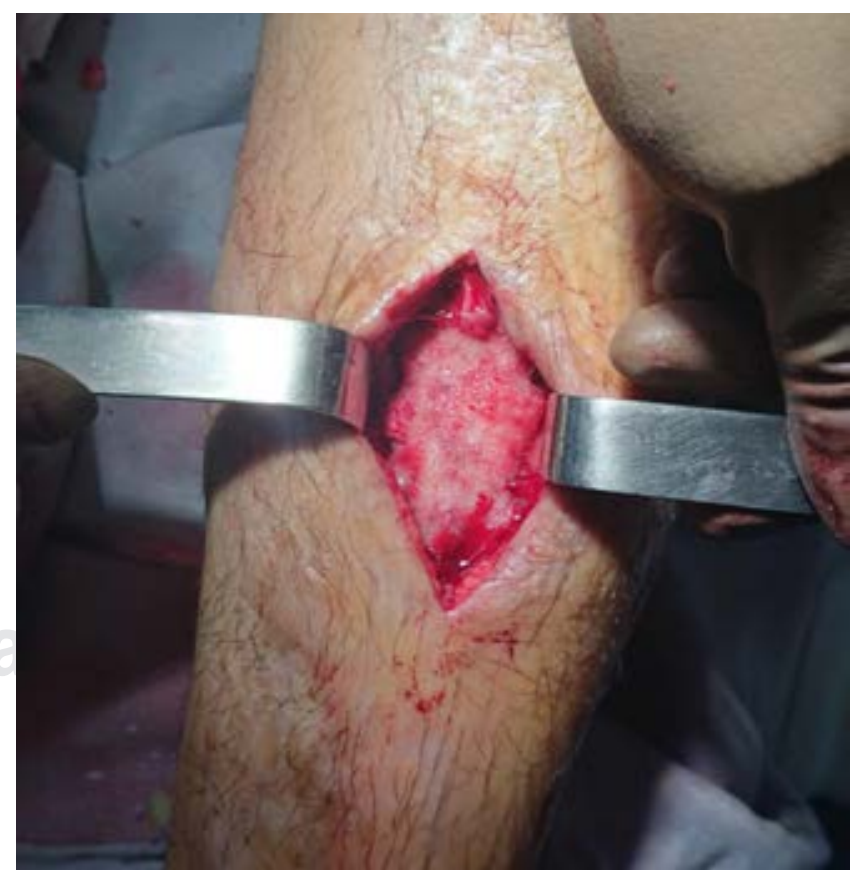

Figura 3: Foto transquirúrgica posterior a colocación de cemento en defecto óseo. 

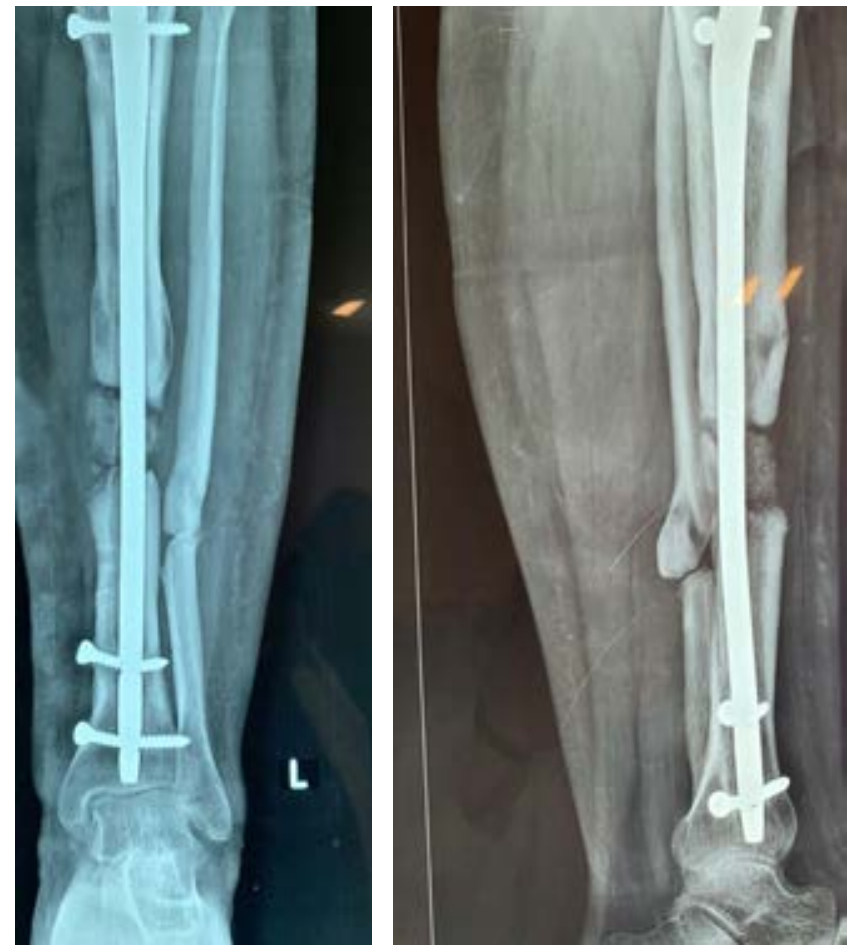

Figura 4: Radiografía seis meses posteriores al inicio del tratamiento con técnica de Masquelet, con datos de consolidación ósea.

\section{Descripción de técnica}

Es de suma importancia preparar al enfermo para una apropiada aplicación de la técnica, evaluar el estado de los tegumentos blandos, realizar las pruebas específicas de laboratorio anticipando diagnosticar o descartar una infección activa en hueso, así como informar del procedimiento al paciente con una adecuada comunicación enfocada a los probables múltiples procedimientos que se necesiten hasta la consolidación del hueso.

Paso 1. En este paso hay que realizar un correcto desbridamiento de los bordes del defecto a tratar, se puede tomar tejido para posterior estudio, si se evidencia la presencia de infección activa en hueso se debe retirar el material previo de osteosíntesis, se debe colocar el espaciador con aglomerante quirúrgico (polimetilmetacrilato), aunque en la práctica original no se describió el uso de cemento medicado, la recomendación actual de antibiótico en 40 $\mathrm{g}$ de tal mezcla son: $4 \mathrm{~g}$ de vancomicina, 0.5 a $3 \mathrm{~g}$ de gentamicina, $2 \mathrm{~g}$ de meropenem o $1 \mathrm{~g}$ de clindamicina. ${ }^{1}$

La estabilización es un proceso clave en este paso, ya sea por fijación interna o externa, dependiendo del estado de los tejidos blandos y de los óseos, si es necesario se puede repetir este paso hasta una adecuada cobertura de dichas zonas o una satisfactoria formación de membrana alrededor del separador de cemento, aquí se optó por la afirmación interna ya que, en la experiencia de los autores, es el mejor implante que cumple con el objetivo de la estabilidad, la finalidad de la creación de tal revestimiento es una cobertura del foco del defecto óseo con tejido vascularizador ideal para la posterior aplicación de injerto. 1,10,11

Paso 2. Al cabo de seis a ocho semanas se retira el espaciador con especial cuidado de mantener la membrana que lo rodea, se recubre el área con injerto ya sea autólogo o heterólogo, se recomienda de cresta iliaca o de canal intramedular con sistema RIA (Reamer-IrrigatorAspirator), si se mantiene una fijación externa, se podría optar por el recambio a colocación de implante, ya sea clavo intramedular o placa; durante esta etapa se pueden añadir recursos osteoinductores u osteoconductores, no obstante, se requieren más estudios antes de recomendar su uso estándar. $1,10,11$

En la paciente descrita se usó un injerto heterólogo, así como matriz ósea, obteniendo resultados funcionales favorables.

\section{Seguimiento}

Se debe tomar evidencia radiográfica cada dos meses, y se permitirá la descarga hasta tener signos de formación de callo en al menos tres corticales del defecto óseo, se ha de individualizar cada caso para el inicio de la descarga, ya sea por auxiliares de la marcha o de forma independiente; sin embargo, se puede hablar de una adecuada estabilización al tener datos radiográficos de consolidación y apoyo sin dolor en la extremidad intervenida.,11

\section{CONCLUSIÓN}

Aunque es una técnica relativamente nueva y que no cuenta con la aprobación total de los cirujanos a escala mundial, sobre todo de aquellos partidarios del método de transporte óseo con llizarov, existen múltiples estudios que confirman los buenos alcances de esta práctica, con porcentajes aceptables de fijación y buena función versus complicaciones, con la ventaja de requerir menor habilidad quirúrgica hacia su realización y con un mejor alcance de los materiales necesarios para la realización de esta técnica en comparación con el transporte óseo. 1,10-12

En la experiencia de los autores, se requiere de pacientes en los que se tenga la certeza de que puedan tener un seguimiento estrecho, así como estar familiarizado con el proceso de la intervención por parte del cirujano, al abrir las membranas del foco de no unión es necesario no retirarlas por completo ni dañarlas, ya que es un paso clave en las consecuencias finales, se deberá cuidar la irrigación buscando no lastimar el tejido circundante. 
Es una técnica con buenos resultados funcionales, que requiere un buen apego por parte del enfermo y el equipo médico tratante, al igual que una adecuada comunicación con el paciente, informándole en todo momento acerca de la posibilidad de múltiples intervenciones hasta la fijación. Aunque existen múltiples variantes del procedimiento en diversos centros a nivel mundial, se trató de ceñirse lo más posible a la original, sin embargo, los autores opinan que la misma se puede perfeccionar con diversos recursos de la ortopedia moderna, como material biológico e inductores de consolidación, por otra parte, se necesitan estudios que avalen esta recomendación.

\section{REFERENCIAS}

1. Azi ML, de Almeida Teixeira AA, Cotias RB, Joeris A, Kfuri M. Inducedmembrane technique in the management of posttraumatic bone defects. JBJS Essential Surgical Techniques. 2019; 9 (2): e22. Available in: https://doi.org/10.2106/jbjs.st.18.00099

2. Masquelet AC. Muscle reconstruction in reconstructive surgery: soft tissue repair and long bone reconstruction. Langenbeck's Arch Sur. 2003; 388 (5): 344-346. Available in: https://doi.org/10.1007/ s00423-003-0379-1

3. Verboket RD, Leiblein M, Janko M, Schaible A, Brune JC, Schroder K et al. From two stages to one: acceleration of the induced membrane (Masquelet) technique using human acellular dermis for the treatment of non-infectious large bone defects. European Journal of Trauma and Emergency Surgery. 2020; 46: 317-327. EJTES. Available in: https:// doi.org/10.1007/s00068-019-01296-x

4. Azi, Matheus L, Teixeira AA de A, Cotias RB, Joeris A, Kfuri M, Jr. Membrane induced osteogenesis in the management of posttraumatic bone defects. J Orthop Trauma. 2016; 30 (10): 545-550. Available in: https://doi.org/10.1097/bot.0000000000000614
5. Hatashita S, Kawakami R, Ejiri S, Sasaki N, Toshiki N, Ito M et al. Acute Masquelet technique' for reconstructing bone defects of an open lower limb fracture. Eur J Trauma Emerg Surg. 2020. Available in: https://doi.org/10.1007/s00068-019-01291-2

6. Mi M, Papakostidis C, Wu X, Giannoudis PV. Mixed results with the Masquelet technique: a fact or a myth? Injury. 2020; 51 (2): 132-135. Available in: https://doi.org/10.1016/j.injury.2019.12.032

7. Roddy E, DeBaun MR, Daoud-Gray A, Yang YP, Gardner MJ. Treatment of critical-sized bone defects: clinical and tissue engineering perspectives. Eur J Orthop Surg Traumatol. 2018; 28 (3): 351-362. Available in: https://doi.org/10.1007/s00590-017-2063-0

8. Liu S, Tao S, Tan J, Hu X, Liu H, Li Z. Long-term follow-up of fibular graft for the reconstruction of bone defects. Medicine. 2018; 97 (40): e12605. Available in: https://doi.org/10.1097/ md.0000000000012605

9. Mathieu L, Bilichtin E, Durand M, de I'Escalopier N, Murison JC, Collombet JM et al. Masquelet technique for open tibia fractures in a military setting. Eur J Trauma Emerg Surg. 2020; 46 (5): 1099-1105. Available in: https://doi.org/10.1007/s00068-019-01217-y

10. Morwood MP, Streufert BD, Bauer A, Olinger C, Tobey D, Beebe M et al. Intramedullary nails yield superior results compared with plate fixation when using the masquelet technique in the femur and tibia. J Orthop Trauma. 2019; 33 (11): 547-552. Available in: https://doi. org/10.1097/bot.0000000000001579

11. Raven TF, Moghaddam A, Ermisch C, Westhauser F, Heller R, Bruckner $\mathrm{T}$ et al. Use of Masquelet technique in treatment of septic and atrophic fracture nonunion. Injury. 2019; 50 (3): 40-54. Available in: https:// doi.org/10.1016/j.injury.2019.06.018

12. Morelli I, Drago L, George DA, Gallazzi E, Scarponi S, Romano CL. Masquelet technique: myth or reality? A systematic review and meta-analysis. Injury. 2016; 47 (6): S68-S76. Available in: https:// doi.org/10.1016/s0020-1383(16)30842-7

Conflicto de intereses: No se reporta conflicto de intereses. 